\title{
Where are the hernias? A paradoxical decrease in emergency hernia surgery during COVID-19 pandemic
}

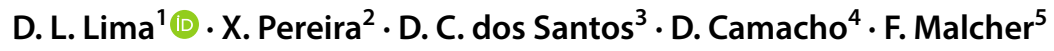

Received: 23 May 2020 / Accepted: 10 June 2020 / Published online: 16 June 2020

๑) Springer-Verlag France SAS, part of Springer Nature 2020

As our hospitals become overwhelmed with the COVID19 pandemic, our ability to provide timely and safe emergency surgical care has been called into question [1] In our hospital, located at the Bronx, NY, in which is located in one of the most affected zip codes in the country, our surgical department was largely re-deployed to non-surgical services to help our overburdened medicine colleagues. Nonetheless, our surgeons remained committed to providing adequate emergency surgical care. Surprisingly, we experienced a decrease in the number of patients presenting with emergency surgical ailments, especially those presenting with incarcerated hernias. This paradoxical effect has been described by other authors and largely attributed to the concept of competing risk, patient apprehension, and secondary effects of an overburdened system [1,2]. At our institution, the first COVID-19 case was admitted on March 11th, 2020. Elective surgeries completely ceased on March 18th, and the peak number of COVID-19 positive patients diagnosed in a day came on April 14, 2020. The total number of emergency cases at the peak of the epidemic, between March 11th and May 10th, was 209, which represents a $48 \%$ reduction in volume when compared to 401 during the same period of 2019.

Looking solely at abdominal wall repairs during this period, we had four emergency cases: two incarcerated inguinal and two ventral hernias (a strangulated umbilical

D. L. Lima

dilaurentino@gmail.com

1 Department of Surgery, Montefiore Medical Center, Bronx, NY, USA

2 Department of Surgery, Montefiore Medical Center, Bronx, NY, USA

3 Pernambuco Health College, Recife, Brazil

4 Department of Surgery, Montefiore Medical Center, Albert Einstein College of Medicine, Bronx, NY, USA

5 Abdominal Wall Program, Department of Surgery, Montefiore Medical Center and Assistant Professor, Albert Einstein College of Medicine, Bronx, NY, USA hernia with bowel resection and one incisional hernia). During the same period in 2019, we had 18 emergency hernia cases; 7 inguinal and 11 ventral repairs, 2 of which incisional hernias. At our hospital, we have a dedicated abdominal wall program that performs roughly 1500 hernia repairs annually. Thus, when elective procedures were cancelled, we expected an increased number of hernia-related surgical emergencies. Yet, we experienced the opposite. The proportion of emergency to elective hernia surgeries in 2020 was significantly lower when compared to the same period of $2019(1.9 \%$ vs $4.9 \%, p=0.03)$. Following a similar trend, consultations for hernia-related pathologies decreased by $66 \%$ during the pandemic.

In recent years, multiple authors have proposed early repair of inguinal hernia to prevent complications such as strangulation and obstruction, which they collective name hernia accidents [3]. A recent meta-analysis showed that an elective repair has a $0.2 \%$ mortality rate, while a hernia accident has a mortality of 4\% [3]. However, randomized controlled trials have showed that a watchful waiting approach (WWA) for high-risk patients is safe with a low incidence of complications [3]. Gong and Li compared the results of eight trials with a WWA and concluded it is safe in the shortterm for asymptomatic or minimally symptomatic inguinal hernias [4]. For ventral hernias, symptoms of pain and risk of strangulation or incarceration are the classic indications for a repair [5]. Only recently have studies started to consider a WWA for specific high-risk patients [5]. Nonetheless, the factors leading to failure of WWA are not clearly defined for either type of hernia (Table 1).

Overall, our institution experienced a drastic reduction of hernia-related emergency cases and number of consultations for hernia-related pathologies during the COVID19 pandemic. Although the decrease in volume is multifactorial, it does highlight a few important considerations of particular interest to hernia specialists. Chiefly, what is the true incidence of hernia-related emergencies requiring prompt surgical attention? It is likely that many patients have 
Table 1 Emergency surgeries in the period from March 11th to May 10th 2019 and 2020 at Montefiore Medical Center

\begin{tabular}{llll}
\hline Emergency surgeries & 2019 & 2020 & $p$ value \\
\hline Hernia repairs-total & 18 & 4 & \\
Inguinal & 7 & 2 & \\
Ventral & 11 & 2 & \\
Total & 401 & 209 & 0.03 \\
$\begin{array}{l}\text { Proportion hernia repairs/ } \\
\text { total cases }\end{array}$ & $4.9 \%$ & $1.9 \%$ & \\
\hline
\end{tabular}

For statistical analysis, the R Project Statistical Computing software, version 3.3.1 (https://www.r-project.org), was used

unknowingly self-selected a WWA given the constraints of the COVID-19 pandemic, revealing a much lower incidence of hernia-related emergencies. If this is the case, are we overusing emergency hernia surgery, which we know comes with inferior results and higher complications rates?

These questions remain difficult to answer, especially with a limited observation over 2 months during a worldwide pandemic, but they bring us back to our initial consideration; where did the hernia-related emergencies go during the COVID-19 pandemic?

Funding There was no funding for this research.

\section{Compliance with ethical standards}

Conflict of interest The authors declare no conflict of interests.
Ethical approval IRB for the approved study (IRB number 202011160).

Statement of human and animal rights The authors declare that the procedures followed were in accordance with the ethical standards of the institutional committee (IRB number 2020-11160) responsible for human experimentations.

Informed consent Informed consent was not applicable.

\section{References}

1. Patriti A, Eugeni E, Guerra F (2020) What happened to surgical emergencies in the era of COVID-19 outbreak? Considerations of surgeons working in an Italian COVID-19 red zone. Update Surg. https://doi.org/10.1007/s13304-020-00779-6

2. Kansagra AP, Goyal MS, Hamilton S, Albers GW (2020) Collateral effect of covid-19 on stroke evaluation in the United States. N Engl J Med. https://doi.org/10.1056/NEJMc2014816

3. Schroeder AD, Tubre DJ, Fitzgibbons RJ (2019) Watchful waiting for inguinal hernia. Adv Surg 53:293-303

4. Gong W, Li J (2018) Operation versus watchful waiting in asymptomatic or minimally symptomatic inguinal hernias: the meta-analysis results of randomized controlled trials. Int J Surg 52:120-125

5. Kokotovic D, Sjølander H, Gögenur I, Helgstrand F (2016) Watchful waiting as a treatment strategy for patients with a ventral hernia appears to be safe. Hernia 20(2):281-287

Publisher's Note Springer Nature remains neutral with regard to jurisdictional claims in published maps and institutional affiliations. 